\title{
Strongly interacting bosons in a one-dimensional optical lattice at incommensurate densities
}

\author{
A. Lazarides ${ }^{1,2}$ O. Tieleman, ${ }^{2}$ and C. Morais Smith ${ }^{2}$ \\ ${ }^{1}$ Max Planck Institute for the Physics of Complex Systems, Noethnitzer Str. 38, D-01187 Dresden, Germany \\ ${ }^{2}$ Institute for Theoretical Physics, Utrecht University, Leuvenlaan 4, NL-3584 CE Utrecht, The Netherlands \\ (Received 8 March 2011; revised manuscript received 7 June 2011; published 16 August 2011)
}

\begin{abstract}
We investigate quantum phase transitions occurring in a system of strongly interacting ultracold bosons in a one-dimensional optical lattice. After discussing the commensurate-incommensurate transition, we focus on the phases appearing at an incommensurate filling. We find a rich phase diagram, with superfluid, supersolid, and solid (kink-lattice) phases. Supersolids generally appear in theoretical studies of systems with long-range interactions; our results break this paradigm and show that they may also emerge in models including only short-range (contact) interactions, provided that quantum fluctuations are properly taken into account.
\end{abstract}

DOI: 10.1103/PhysRevA.84.023620

PACS number(s): 03.75.Hh, 05.30.Jp

\section{INTRODUCTION}

The rapid progress in trapping and cooling atoms has rendered the study of "tailor-made" low-dimensional systems [1] experimentally accessible. Both the dimensionality and the interactions can be controlled, allowing great flexibility in realizing almost arbitrary strongly correlated physical systems. A superfluid-Mott-insulator (SF-MI) quantum phase transition, driven by increasing the potential depth of the optical lattice (and hence the relative strength of interactions) beyond a critical value, has been observed for bosons loaded into an optical lattice in three [2], two [3], and one [4] dimensions. In addition, the Tonks-Girardeau gas, where bosons avoid spatial overlap and acquire fermionic properties due to strong repulsive interactions, has been experimentally realized in one dimension (1D) [5].

Recently, a new type of quantum phase transition was observed in $1 \mathrm{D}$ in the very strongly interacting regime: for an arbitrarily weak optical lattice potential commensurate with the atomic density of the Bose gas, a quantum phase transition into an insulating, gapped state was observed with the atoms pinned at the lattice minima [6]. Theoretical studies of onedimensional systems based on the sine-Gordon model indeed predict that above a critical interaction strength, the superfluid (SF) phase should become a MI even for a vanishingly weak optical lattice [7].

In this article, we show that another interesting regime can be reached if the density is incommensurate with the optical lattice. The system is then described by a driven sineGordon model. In this model, the appearance of superfluidity (off-diagonal correlations) may be driven in two different ways, either by tuning the interaction strength at constant lattice depth and commensurate period, as already realized experimentally [6], or by tuning the density or lattice parameter away from commensurability. We study the excitations of the system in the incommensurate phase and show that a supersolid (SS) phase may arise. In addition, for sufficiently large lattice strengths, a solid (S) phase is stabilized even at nonunit filling.

Our approach to studying this system is as follows: First, following previous work [6-8], we formulate the underlying one-dimensional interacting boson problem in terms of a quantum sine-Gordon field theory, with the deviation of the number density from commensurate values driving the appearance of kinks in the field. We carefully obtain the density threshold for the commensurate-incommensurate SFMI transition, finding that the SF appears for arbitrarily small deviations of the density from the commensurate density, in agreement with Ref. [9].

We next study the incommensurate regime, in which the excess particles appear as kinks of the sine-Gordon field, having an effective mass and effective interactions different from the bare particles. We extract these two parameters exactly from the underlying field theory and finally apply a functional renormalization group (RG) approach to the path integral formulation of the many-body statistical density matrix to obtain the ground-state properties of the system. The RG transformation shows that quantum fluctuations renormalize the interactions between the kinks to a power law form; this maps the system to the Calogero-Sutherland model [10], an exactly solvable model of one-dimensional particles with long-range interactions. This finally allows us to propose a phase diagram for the incommensurate regime (see Fig. 1). When the lattice potential is strong enough, the system solidifies. However, the $\mathrm{S}$ phase here is actually a lattice of kinks, and the number of particles per site is not fixed. At intermediate values of the lattice potential, we predict the emergence of a SS phase. SS phases usually occur in model Hamiltonians which include long-range interactions, and have a characteristic wavelength which is an integer multiple of the lattice spacing [11]. The most striking feature of the system studied in this article is that while the original Hamiltonian contains only local interactions, the SS phase appears due to the finite-range nature of the interaction between the excitations. In addition, the periodicity of the SS phase found here is unrelated to that of the lattice, a qualitatively different behavior from the situations usually found in the literature.

\section{MICROSCOPIC MODEL}

The microscopic description of a trapped gas of cold bosons in 1D with contact interactions and in the presence of a singleparticle potential $V(x)$ is

$$
\begin{aligned}
H= & \int_{-\infty}^{\infty} d x\left\{\psi^{\dagger}(x)\left(-\frac{\hbar^{2}}{2 m} \nabla^{2}+V(x)\right) \psi(x)\right. \\
& \left.+\frac{g}{2} \int_{-\infty}^{\infty} d x \psi^{\dagger}(x) \psi^{\dagger}(x) \psi(x) \psi(x)\right\}
\end{aligned}
$$




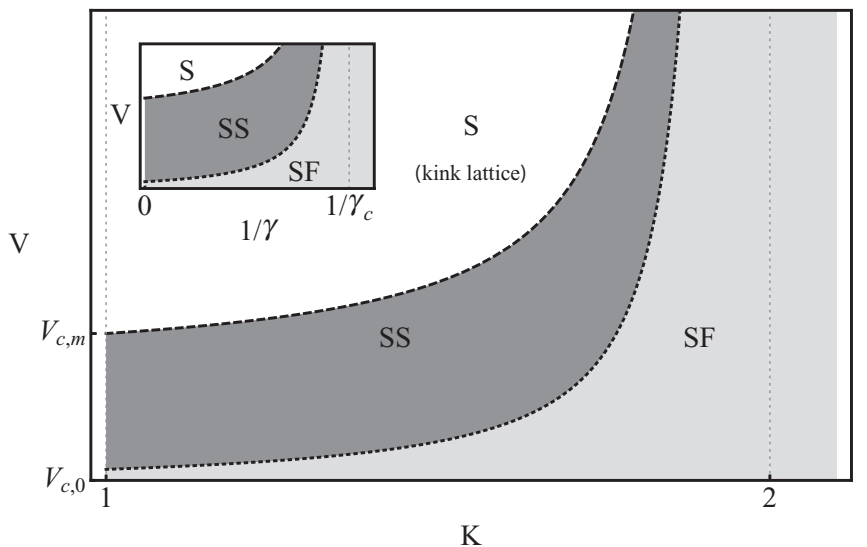

FIG. 1. $T=0$ phase diagram for a filling factor slightly above unit filling. SF, SS, and S indicate superfluid, supersolid and solid phases, respectively. The inset shows the same phase diagram but in terms of $\gamma$, the dimensionless interaction strength, instead of the Tomonaga-Luttinger parameter $K$.

where $g$ is the strength of the $\delta$-function interaction, $\psi\left(\psi^{\dagger}\right)$ are bosonic annihilation (creation) operators, and $m$ is the atomic mass. The parameter characterizing the strength of the interactions is the Lieb-Liniger parameter $\gamma=m g / \hbar^{2} n_{0}$, where $n_{0}$ is the average density.

Writing $\psi(x)=\sqrt{n(x)} \exp (-i \theta(x))$, with $n(x)$ the density and $\theta(x)$ the (real) phase, and using the Poisson summation formula, the density operator may then be expressed as $[7,8]$

$$
n(x)=\left[n_{0}-\frac{1}{\pi} \partial_{x} \phi(x)\right] \sum_{p=-\infty}^{\infty} e^{2 i p\left[\pi n_{0} x-\phi(x)\right]},
$$

where $\int d x \partial_{x} \phi(x)=0$. Equation (2) yields an expression for the bosonic operators in Eq. (1) in terms of the new field $\phi$. The appropriate bosonic commutation relations are satisfied if $\left[\partial_{x} \phi(x), \theta\left(x^{\prime}\right)\right]=-i \pi \hbar \delta\left(x-x^{\prime}\right)$, that is, if $\theta$ and $\partial_{x} \phi / \pi$ are canonically conjugate variables. From Eq. (2), it follows that kinks in the $\phi$ field correspond to particlelike excitations. This fact will be of great importance to us later on.

In the long-wavelength limit, and in the presence of an optical lattice creating a single-particle potential $V(x)=$ $(V / 2) \cos \left(4 \pi x / \lambda_{L}\right)$, the system of Eq. (1) may be described by an action of the form

$$
\begin{aligned}
\mathcal{S}[\phi]= & \int_{0}^{\beta} d \tau \int_{-\infty}^{\infty} d x \frac{1}{8 \pi K}\left[\left(\partial_{x} \phi\right)^{2}+\left(\partial_{\tau} \phi\right)^{2}\right] \\
& +\frac{1}{2} u \int_{0}^{\beta} d \tau \int_{-\infty}^{\infty} d x \cos [\phi(x)-Q x],
\end{aligned}
$$

where we have now set $\hbar=1$, scaled lengths such that the speed of sound is unity, and finally scaled $\phi \rightarrow \phi / 2$. Here, $\beta=$ $1 / k_{B} T$ and $u=n_{0} V$, while $K$ is the usual Luttinger liquid parameter. For bosons interacting via contact potentials, $K$ may be expressed in terms of $\gamma$; for large $\gamma, K \approx(1+2 / \gamma)^{2}$, while for smaller interaction strengths, $\gamma$ it is given by $K \approx \pi / \sqrt{\gamma-\gamma^{3 / 2} /(2 \pi)}$. Notice that $K \geqslant 1$, as it should for bosons with local interactions. We have also only kept the most relevant (least quickly oscillating in space) terms and written $Q=2 \pi\left(n_{0}-2 / \lambda_{L}\right)$ as the deviation of the average density from its commensurate value.

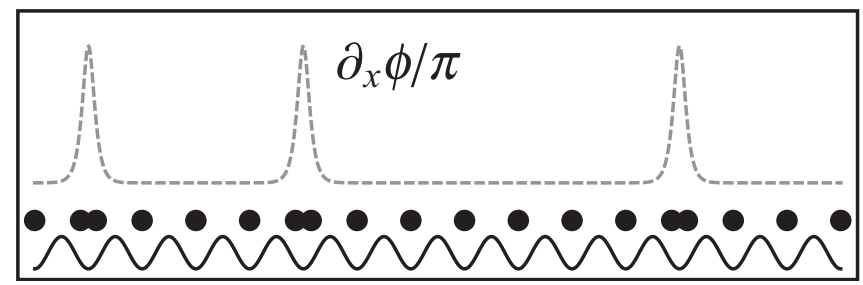

FIG. 2. $Q>0$; the dashed line is $\partial_{x} \phi / \pi$, the solid line is the periodic potential, and the corresponding particle positions are indicated by dots. Notice how kinks (indicated by localized deviations from a straight line for $\partial_{x} \phi / \pi$ ) correspond to particles in excess of the commensurate particle density.

In the zero-temperature, $\beta \rightarrow \infty$ limit, Eq. (3) is formally equivalent to the model studied in Ref. [12]. It is also related to previous work on quantum Hall bilayer systems [13,14], with the important difference that the boundary conditions in the present case are $\int d x \partial_{x} \phi=0$, while in Refs. [12,15] (amongst numerous others), there is no such restriction on $\phi$. This is crucial to the position of the boundary of the commensurateincommensurate transition and is due to the fact that we are working at fixed particle number [9].

Since kinks correspond to excess particles above the commensurate density (see Fig. 2), fixing the particle density must fix the number density of kinks uniquely. But from its definition, $Q$ is directly proportional to this excess particle density, so that the kink density must be proportional to $Q$ itself. This immediately implies that $Q_{c}=0$, at least at zero temperature. ${ }^{1}$ Mathematically, this is a consequence of the boundary condition at the edges of the system, which implies that the commensurate phase cannot exist unless $Q=0$. For any $Q>0$, a finite density of bosonic kinks appears.

\section{EFFECTIVE MODEL FOR EXCITATIONS}

The full analytical expression for the bare interaction between two kinks in a sine-Gordon model is given in Ref. [16]; its limiting forms are

$$
V_{\text {bare }}(r) \rightarrow \begin{cases}\frac{8}{\pi \xi} \exp (-r / \xi), & r \gg \xi \\ \pi /(2 r), & r \ll \xi .\end{cases}
$$

The effective width of the kinks is $\xi=1 / \sqrt{2 \pi K n_{0} V}$, so that this potential amounts to an impenetrable core plus a finiterange repulsion.

The effective kink (and antikink) mass $\sigma$ is strongly renormalized by quantum fluctuations [17]. It may be obtained using a flow-equation RG scheme as described in Ref. [18]; the salient points are that it is proportional to $u$ at $K=1$ (that is, it is not renormalized) and vanishes as $K \rightarrow 2^{-}$. This vanishing of $\sigma$ is also responsible for the SF-MI transition observed for weak lattices in Ref [6] at commensurability; $K \rightarrow 2$ corresponds to a critical $\gamma_{c} \approx 3.5$. We obtain $\sigma$ for intermediate values $1<K<2$ by direct numerical integration of the flow equations given in Ref. [18].

\footnotetext{
${ }^{1}$ In Ref. [7], a finite value for $Q_{c}$ is obtained which, however, should be interpreted as a finite chemical potential, not density.
} 
To study the system of interacting kinks, we employ the statistical density matrix in imaginary time and position representation $[19,20]$. This is given by

$$
\rho(R, R ; \beta)=\int \prod_{j} \mathbb{D} x_{j} \exp \left(-S\left[\left\{x_{n}(\tau)\right\}\right] / \hbar\right),
$$

where $\left\{x_{n}(\tau)\right\}$ denotes the set of positions of the particles at time $\tau, R=\left\{r_{1}, r_{2}, \ldots, r_{N}\right\}$ denotes the set of positions of the particles at $\tau=0$ and $\tau=\beta$ (see below), while $\mathbb{D} x_{j}$ denotes functional integration over $x_{j}$; finally,

$$
S\left[\left\{x_{n}(\tau)\right\}\right]=\frac{1}{2} \int d \tau\left[\sigma \sum_{n}\left(\partial_{\tau} x_{n}\right)^{2}+\sum_{n, m} V\left(x_{n}-x_{m}\right)\right] .
$$

In Eq. (6), the integral runs from zero to $\beta$, and there is an ultraviolet cutoff $\Lambda_{\tau}=2 \pi / \Delta \tau$ with $\Delta \tau$ a discretization step size [20]. In this picture, the world lines of the particles $x_{i}(\tau)$ correspond to classical strings without overhangs, the ends of which are fixed to $x_{i}(0)=x_{i}(\tau)=r_{i}$. Note that Eqs. (5) and (6) describe the $\rho$ appropriate for distinguishable particles; for bosonic particles, one symmetrizes in the end, so that $\rho_{B}\left(R, R^{\prime} ; \beta\right)=\sum_{P} \rho\left(R, P R^{\prime} ; \beta\right)$, with $P$ labeling the permutation.

We begin by estimating the temperature dependence of the critical incommensurability $Q_{d}$ above which exchange effects become important. The world lines of the particles are of length $\beta$ in the timelike direction, and the "width" of the path in the spacelike direction will be $w \propto \sqrt{\hbar^{2} \beta / \sigma}$. If the average interkink distance, proportional to $Q^{-1}$, is larger than this, quantum effects are not important; the condition for the statistics to be important is, therefore, $Q \sqrt{\hbar^{2} \beta / \sigma}>1$, up to a numerical factor. This defines a critical $Q_{d} \propto \sqrt{k_{B} T \sigma / \hbar^{2}}$. Below this $Q_{d}$, the kinks behave like noninteracting bosons; above it, they begin to interact, and we expect the effects described below to be evident. Furthermore, since $\sigma$ vanishes on the lines $V=0$ and $K=2, Q_{d}$ also vanishes there.

\section{PHASE DIAGRAM VIA THE RENORMALIZATION GROUP}

Next, we concentrate on the $T=0$ or $\beta \rightarrow \infty$ limit, corresponding to infinitely long strings; in this limit, the degeneracy condition is always satisfied. We shall employ a renormalization group $(\mathrm{RG})$ technique applied directly to the density matrix of Eq. (5). Details of this will be presented elsewhere [21]; here, we shall only outline our conclusions.

Splitting the fields $x_{i}$ into slow and fast parts as usual [22], it is possible to extend the Wegner-Houghton approach [23,24] to the many-body case, obtaining the flow equation for the potential

$$
\partial_{\epsilon} V=V+\frac{1}{2} x \partial_{x} V+\hbar \Lambda_{\tau} \log \left(1+\frac{2}{\sigma \Lambda_{\tau}} \partial_{x}^{2} V\right) .
$$

Notice that the coarse graining is done in the $\tau$ direction; thus, information on length scales comparable to the kink density is still present in the fixed point potentials.

The physics of the system is determined by the fixed point potentials of Eq. (7). For bare (initial) potentials that diverge at the origin, ${ }^{2}$ these may be determined numerically; for $x \gg$ $\hbar / \Lambda_{\tau} \sigma$, their behavior is

$$
V_{\mathrm{FP}}=\frac{\hbar^{2}}{2 \sigma} \frac{\lambda(\lambda-1)}{x^{2}},
$$

where we have written the coefficient of $x^{-2}$ as $\hbar^{2} \lambda(\lambda-1) / 2 \sigma$ in order to make contact with the conventional notation in the literature (see below). We are, thus, dealing with a system of bosons interacting via an inverse square power law; this is the celebrated Calogero-Sutherland model [10], the groundstate wave function and low-energy spectrum of which are known. We concentrate here on its ground-state properties, which have been studied using numerical techniques [25]. The authors of Ref. [25] find (quasi-)long-range off-diagonal order for $0<\lambda<2$, while they find (quasi-)long-range diagonal order for $\lambda>1$. The system is, thus, in a condensed, SF state for $0<\lambda<1$, in a SS state, characterized by the simultaneous presence of diagonal and off-diagonal long-range order for $1<\lambda<2$, and in a crystalline, $\mathrm{S}$ state characterized by strong diagonal correlations for $2<\lambda$. Therefore, the phase in which the system is for incommensurate densities $(Q \neq 0)$ depends on the range in which the $\lambda$ corresponding to the potential in Eq. (4) lies.

To map out the phase diagram, we note that local analysis of the fixed point ordinary differential equation, Eq. (7) with the left-hand side set to 0 , indicates that the fixed point potentials, $V_{\mathrm{FP}}$, have the property that $\partial V_{\mathrm{FP}} / \partial \sigma>0$ (for all $x$ ). An increase in $\sigma$, therefore, results in an increase in $\lambda$ of Eq. (8). In addition, at $K=1$ and $V=0$ (hard-core free bosons), $\lambda=1$ [26]. Based on these two pieces of information, and the behavior of the mass described earlier, we propose the phase diagram in Fig. 1 for $T=0$. Starting from the point $K=1, V=0$, an increase of $V$ causes a rapid increase of $\sigma$, which corresponds to an increase in $\lambda$ so that $\lambda>1$ which corresponds to a SS phase. As $V$ is further increased, $\lambda$ reaches the value $\lambda=2$ at $V=V_{c, m}$, at which point phase coherence is lost, the structure factor displays a sharp peak [25], and the system is in the $\mathrm{S}$ state. On the other hand, starting from any point on the $K=1$ line and increasing $K$ corresponds to decreasing $\sigma$, thus decreasing $\lambda$ from its value at $K=1$. As a result, the line $V_{c, m}$ curves upward as $K$ increases. Starting from $V_{c, 0}(K=1)=0$ and increasing $K, \lambda$ must decrease below 1 so that the diagonal order is suppressed; thus, the line $V_{c, 0}$ separating the $\mathrm{SF}$ from the $\mathrm{SS}$ region also curves upward. As $K \rightarrow 2$, or $\gamma \rightarrow 3.5$, the effective mass vanishes for any $V$; this results in a rapid decrease of $\lambda$, so that both lines curve upward sharply.

It is important to note that the presence of the SS phase represents an order out of disorder effect: quantum fluctuations, which at first sight one would expect disorder the system, result in a strengthening of the repulsion which in turn causes the system to order.

Let us briefly discuss the differences between the phases just described in terms of experimentally accessible quantities. The

\footnotetext{
${ }^{2}$ Note that the potential between a kink and an antikink does not diverge at the origin [16]; thus, our analysis does not apply to the SF obtained at $K>2$.
} 
main distinguishing features of these phases are the diagonal and off-diagonal correlations. Off-diagonal long-range order may be observed using time-of-flight measurements, which, therefore, allow us to distinguish the SF and SS phase-coherent phases from the $\mathrm{S}$ phase; in the latter, phase correlations drop quickly and the time-of-flight image is smeared. On the other hand, techniques for measuring density variations would distinguish between the SS and S phases on one hand and the SF phase on the other; single-site addressability is possible [27], which may allow us to detect density oscillations.

We emphasize that the phase diagram of Fig. 1 is obtained by considering the quasiparticles (kinks) and not the underlying bosons; in effect, these form a new interacting system which we study using our functional RG flow equation.

\section{CONCLUSIONS}

In summary, we have shown that the incommensurabilityinduced SF-MI transition occurs for arbitrarily small incommensurability. We have then studied the system of bosonic quasiparticles which appears as soon as commensurability is lost; calculating the effective interactions between them, as well as their effective mass, and using an RG transformation, we have argued that quantum fluctuations drive the interactions to acquire an inverse square form (a Calogero-Sutherland model). A phase diagram for the current system of strongly interacting bosons in a weak one-dimensional optical lattice is then proposed, which features SF, SS, and S phases. The most striking feature of our calculations is the appearance of a SS phase, in spite of the local character of the interactions in the original model. The periodicity of both the SS and the S phases is unrelated to that of the underlying lattice, thus providing us with more exotic states of matter.

Although the experimental setup of Ref. [6] allows us, in principle, to tune the density and to investigate also the commensurate-incommensurate quantum phase transition, up to now only the commensurate regime has been studied. Our studies indicate that the elusive SS phase is within reach by modifying a single parameter in the experimental setup of Ref. [6]. We hope that our work will trigger further experiments into this fascinating and largely unexplored regime.

\section{ACKNOWLEDGMENTS}

We acknowledge financial support from the Netherlands Organization for Scientific Research (NWO), and thank J.-S. Caux, G. Japaridze, and L. K. Lim for stimulating discussions and for a critical reading of the manuscript.
[1] M. A. Cazalilla, R. Citro, T. Giamarchi, E. Orignac, and M. Rigol, e-print arXiv:1101.5337.

[2] M. Greiner et al., Nature (London) 415, 39 (2002).

[3] I. B. Spielman, W. D. Phillips, and J. V. Porto, Phys. Rev. Lett. 98, 080404 (2007).

[4] T. Stöferle, H. Moritz, C. Schori, M. Kohl, and T. Esslinger, Phys. Rev. Lett. 92, 130403 (2004).

[5] B. Paredes et al., Nature (London) 429, 277 (2004); T. Kinoshita et al., Science 305, 1125 (2004).

[6] E. Haller et al., Nature (London) 466, 597 (2010).

[7] H. P. Buchler, G. Blatter, and W. Zwerger, Phys. Rev. Lett. 90, 130401 (2003).

[8] F. D. M. Haldane, Phys. Rev. Lett. 47, 1840 (1981).

[9] T. Giamarchi, Quantum Physics in One Dimension (Oxford University Press, Oxford, 2004).

[10] B. Sutherland, Beautiful Models (World Scientific, Singapore, 2004).

[11] T. Lahaye et al., Rep. Prog. Phys. 72, 126401 (2009); P. Sengupta, L. P. Pryadko, F. Alet, M. Troyer, and G. Schmid, Phys. Rev. Lett. 94, 207202 (2005); I. Danshita and C. A. R. Sa de Melo, ibid. 103, 225301 (2009); O. Tieleman, A. Lazarides, and C. Morais Smith, Phys. Rev. A 83, 013627 (2011); L. Mathey, I. Danshita, and C. W. Clark, ibid. 79, 011602 (2009).

[12] A. Lazarides, O. Tieleman, and C. Morais Smith, Phys. Rev. B 80, 245418 (2009).

[13] O. Tieleman, A. Lazarides, D. Makogon, and C. Morais Smith, Phys. Rev. B 80, 205315 (2009).
[14] N. Read, Phys. Rev. B 52, 1926 (1995).

[15] V. L. Pokrovsky and A. L. Talapov, Phys. Rev. Lett. 42, 65 (1979); M. E. Fisher and D. S. Fisher, Phys. Rev. B 25, 3192 (1982); G. I. Japaridze and A. A. Nersessyan, JETP Lett. 27, 335 (1978).

[16] J. K. Perring and T. H. R. Skyrme, Nucl. Phys. 31, 550 (1962).

[17] A. O. Gogolin, A. A. Nersesyan, and A. M. Tsvelik, Bosonization and Strongly Correlated Systems (Cambridge University Press, Cambridge, 1998).

[18] S. Kehrein, Phys. Rev. Lett. 83, 4914 (1999); Nucl. Phys. B 592, 512 (2001).

[19] R. P. Feynman and A. R. Hibbs, Quantum Mechanics and Path Integrals (McGraw-Hill, New York, 1965).

[20] D. M. Ceperley, Rev. Mod. Phys. 67, 279 (1995).

[21] A. Lazarides (unpublished).

[22] S. K. Ma, Modern Theory of Critical Phenomena (AddisonWesley, California, 1976).

[23] F. J. Wegner and A. Houghton, Phys. Rev. A 8, 401 (1973).

[24] R. Lipowsky and M. E. Fisher, Phys. Rev. B 36, 2126 (1987).

[25] G. E. Astrakharchik, D. M. Gangardt, Y. E. Lozovik, and I. A. Sorokin, Phys. Rev. E 74, 021105 (2006).

[26] M. A. Cazalilla, J. Phys. B 37, S1 (2004).

[27] T. Gericke et al., Nat. Phys. 4, 949 (2008); W. S. Bakr et al., Science 329, 547 (2010); N. Gemelke et al., Nature (London) 460, 995 (2009); J. F. Sherson et al., ibid. 467, 68 (2010). 